\title{
The role of surgical clips in the evaluation of interfractional uncertainty for treatment of hepatobiliary and pancreatic cancer with postoperative radiotherapy
}

\author{
Jin Suk Bae, MD', Dong Hyun Kim, MD ${ }^{1,2}$, Won Taek Kim, MD ${ }^{1,2}$, Yong Ho Kim, MS ${ }^{1}$, Dahl Park, PhD ${ }^{1,2}$, \\ Yong Kan Ki, MD ${ }^{1,2}$ \\ ${ }^{1}$ Department of Radiation Oncology, ${ }^{2}$ Biomedical Research Institute, Pusan National University Hospital, \\ Pusan National University School of Medicine, Busan, Korea
}

Purpose: To evaluate the utility of implanted surgical clips for detecting interfractional errors in the treatment of hepatobiliary and pancreatic cancer with postoperative radiotherapy (PORT).

Methods and Materials: Twenty patients had been treated with PORT for locally advanced hepatobiliary or pancreatic cancer, from November 2014 to April 2016. Patients underwent computed tomography simulation and were treated in expiratory breathing phase. During treatment, orthogonal kilovoltage (kV) imaging was taken twice a week, and isocenter shifts were made to match bony anatomy. The difference in position of clips between $\mathrm{kV}$ images and digitally reconstructed radiographs was determined. Clips were consist of 3 proximal clips (clip_p, $\leq 2 \mathrm{~cm}$ ) and 3 distal clips (clip_d $>2 \mathrm{~cm}$ ), which were classified according to distance from treatment center. The interfractional displacements of clips were measured in the superior-inferior (SI), anterior-posterior (AP), and right-left (RL) directions.

Results: The translocation of clip was well correlated with diaphragm movement in 90.4\% (190/210) of all images. The clip position errors greater than $5 \mathrm{~mm}$ were observed in $26.0 \%$ in $\mathrm{SI}, 1.8 \%$ in AP, and $5.4 \%$ in RL directions, respectively. Moreover, the clip position errors greater than $10 \mathrm{~mm}$ were observed in $1.9 \%$ in $\mathrm{SI}, 0.2 \%$ in $\mathrm{AP}$, and $0.2 \%$ in RL directions, despite respiratory control.

Conclusion: Quantitative analysis of surgical clip displacement reflect respiratory motion, setup errors and postoperative change of intraabdominal organ position. Furthermore, position of clips is distinguished easily in verification images. The identification of the surgical clip position may lead to a significant improvement in the accuracy of upper abdominal radiation therapy.

Keywords: Postoperative radiotherapy, Clip, Hepatobiliary cancer, Pancreatic cancer, Interfractional error

\section{Introduction}

During upper abdominal irradiation, internal organs move unexpectable directions. Major factors of treatment uncertainty are setup error, breathing motion and peristalsis. These organ motions lead to treatment errors and inadequate dose delivery. As a result, it requires wide margins to compensate inadequate dose distribution [1]. Probability of toxicity increases with wide

Received 9 November 2016, Revised 26 December 2016, Accepted 10 January 2017.

Correspondence: Won Taek Kim, MD, Department of Radiation Oncology, Pusan National University Hospital, Pusan National University School of Medicine, 179 Gudeok-ro, Seo-gu, Busan 49241, Korea. Tel: +82-51-240-7383, Fax: +82-51-2485747, E-mail: rokwt@hanmail.net

(c) This is an Open Access article distributed under the terms of the Creative Commons Attribution Non-Commercial License (http://creativecommons.org/ licenses/by-nc/4.0/) which permits unrestricted non-commercial use, distribution, and reproduction in any medium, provided the original work is properly cited.

www.e-roj.org 
safety margin. Clinical target volume (CTV) usually has been expanded 5-10 $\mathrm{mm}$ to obtain a planning target volume (PTV). Although studies have examined the amount of margin needed to adequately cover a volume in relatively static sites such as the head and neck region, the same undertaking has not been reported for more dynamic sites such as the abdomen [2]. During treatment, organ motion can lead to excursions outside the designated PTV margin, resulting in underdosing of the CTV.

Clinical study to quantify the intrafraction movement of pancreas, liver and kidneys showed a substantial movement of the upper abdominal organs mainly due to respiration [3]. Respiratory organ motion during irradiation can be reduced with some respiratory motion control methods such as abdominal compression, breath holding, active breathing control, respiratory gated beam delivery, dynamic tumor tracking, and so on. Use of image-guided radiation therapy improves treatment accuracy.

Bowel position change and delayed gastric emptying caused by surgical procedures are additional concern for upper abdominal tumor [4]. In this situation, artificial indicators can serve as surrogate markers for organ position.

Implantation of gold fiducial markers for stereotactic body radiation therapy for the treatment of the lung or liver cancer has possibility of additional risk of invasive procedure [5]. Postoperative surgical clips have the advantage of noninvasiveness. Moreover, metallic surgical clips were detected easily on kilovoltage $(\mathrm{kV})$ verification images. Surgical clip displacement reflect postoperative change of intra-abdominal organ position as well as respiratory motion. In this study, we quantified surgical clip shift and evaluated the utility of implanted surgical clips for detecting interfractional errors in the treatment of hepatobiliary and pancreatic cancer with postoperative radiotherapy (PORT).

\section{Materials and Methods}

Twenty locally advanced hepatobiliary and pancreatic cancer patients treated with postoperative concurrent chemoradiotherapy or PORT from November 2014 to April 2016 were included in this study. The characteristics of patients are described in Table 1. The study protocol was performed with the approval from the Institutional Review Board (IRB) of Pusan National University.

\section{Simulation and planning}

Patients underwent computed tomography (CT) simulation
Table 1. Patients' characteristics

\begin{tabular}{lc}
\hline \multicolumn{1}{c}{ Characteristic } & $\begin{array}{c}\text { No. of } \\
\text { patients }\end{array}$ \\
\hline Age (yr), median (range) & $62(49-75)$ \\
Sex & \\
Male & 17 \\
Female & 3 \\
Diagnosis & \\
Cholangiocarcinoma & 9 \\
Intrahepatic & 1 \\
Hilar & 2 \\
Distal & 6 \\
Pancreatic cancer & 6 \\
Ampulla of Vater cancer & 3 \\
Gallbladder cancer & 1 \\
Stomach cancer & 1 \\
Surgical technique & \\
Pylorus-preserving PD & 8 \\
Liver resection, bile duct resection & 6 \\
Whipple's operation & 4 \\
Cholecystectomy & 1 \\
Subtotal stomach-preserving PD/SMV resection & 1 \\
\hline
\end{tabular}

$\mathrm{PD}$, pancreaticoduodenectomy; SMV, superior mesenteric vein.

with intravenous contrast in the supine position, with their arms above the head. The immobilization device was a vacuum bag or custom cast. The patients underwent a training session to hold their breath for at least 20 seconds while under fluoroscopic visualization. Thereafter, 3-mm thickness CT scans were obtained from the level of the carina to the level of iliac crest in expiratory phase. The patients followed routine dietary guidelines before $\mathrm{CT}$ simulation for treatment planning and before each treatment fraction, including fasting for at least 8 hours.

The CTV included tumor bed, intestinal stump, resection margin, anastomosis site and regional lymphatics. One radiation oncologist contoured the CTV and PTV. Expanding margin was 5-10 $\mathrm{mm}$ from CTV to PTV. All plans were generated in Eclipse treatment planning system (Varian Medical Systems, Palo Alto, CA, USA).

\section{Surgical clips}

The difference in position of clips between $\mathrm{kV}$ images and digitally reconstructed radiographs (DRRs) was determined. One radiation oncologist delineated 6 clips on planning CT for each patient (Fig. 1). The surgical clips consist of 3 proximal clips (clip_p, $\leq 2 \mathrm{~cm}$ ) and 3 distal clips (clip_d, $>2 \mathrm{~cm}$ ), which were classified according to distance from treatment center. Proximal area clips (clip_p) included clips in primary tumor 


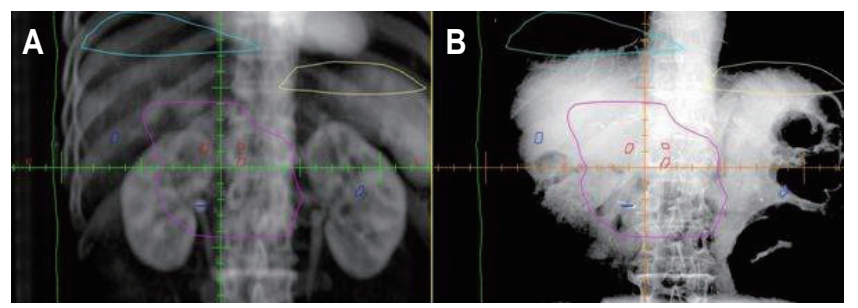

Fig. 1. The location of 6 clips in digitally reconstructed radiographs (A) and kilovoltage images (B). Clips were consist of 3 proximal clips (red, clip_p, $\leq 2 \mathrm{~cm}$ ) and 3 distal clips (blue, clip_d, $>2 \mathrm{~cm}$ ), which were classified according to distance from treatment center. The magenta, sky, and yellow lines show the planning target volume contours, right diaphragm, and left diaphragm, respectively.

bed sites or anastomosis sites, and distal area clips (clip_d) encompassed clips in node dissection sites in general. Easily distinguished clips were preferably selected and contoured. The nearest clip from treatment center was selected in conglomerated clips. Selected clips were placed within $20 \mathrm{~cm}$ $\times 20 \mathrm{~cm}$ distance from treatment center on anterior-posterior (AP) and lateral kV images.

\section{Verification and beam delivery}

Prescribed dose levels ranged from 45.0 to $50.4 \mathrm{~Gy}$ at $1.8 \mathrm{~Gy}$ per fraction for about 5-6 weeks. Body weight and abdominal circumference of each patient were measured daily. Threedimensional conformal radiotherapy was carried out using 6-MV or 15-MV photon beams of linear accelerator (Clinac iX System, Varian Medical Systems). Orthogonal kV images (both AP and lateral views) equipped in linear accelerator were taken twice a week during treatment. The highest point of diaphragm in fluoroscopic image and DRRs was used to determine whether the respiratory phase was controlled. We reminded patient of respiratory instruction before each treatment. Bony markers were aligned to isocenter before each treatment fraction. The clip shifts were measured along three axes: superior (+)-inferior (-), SI; anterior (+)-posterior (-), AP; and right (+)-left (-), RL.

The van Herk margin formula (VHMF) [6] that equation of using systemic error and random error was used to obtain the CTV-PTV margins in all directions:

Margin $=(2.5 \times S D$ of the group systematic error $)+(0.7 \times$ $S D$ of the random error).

\section{Evaluation and statistical analysis}

A total of 210 images were analyzed retrospectively. All clip displacement was calculated after bony alignment. The mean, standard deviation (SD) and shifting range of clip displacements for each patient were calculated. Analyses were performed using SPSS ver. 22 (IBM Inc., Armonk, NY, USA).

\section{Results}

Daily measured body weight and abdominal circumference had changed less than $5 \%$ in all patients. The number of detectable surgical clips in verification image for each patient ranged from 10 to 20. Distance of clips from the treatment center ranged from 0 to $10 \mathrm{~cm}$. The mean shift using bony anatomy was $-0.11 \pm 1.46 \mathrm{~mm}$ (range, -7 to $6 \mathrm{~mm}$ ) in vertical direction, $-0.06 \pm 2.31 \mathrm{~mm}$ (range, -8 to $7 \mathrm{~mm}$ ) in longitudinal direction and $0.16 \pm 2.14 \mathrm{~mm}$ (range, -9 to $6 \mathrm{~mm}$ ) in lateral direction. In this study, 155 of 210 images (73.8\%) require additional shift after bony alignment.

The mean shift to clips after bony alignment was $0.46 \pm 2.66$ $\mathrm{mm}$ (clip_p: range, -15 to $20 \mathrm{~mm}$ ) and $1.23 \pm 3.87 \mathrm{~mm}$ (clip_d: range, -61 to $17 \mathrm{~mm}$ ) in SI, $0.49 \pm 1.26 \mathrm{~mm}$ (clip_p: range, -8 to $6 \mathrm{~mm}$ ) and $1.25 \pm 1.79 \mathrm{~mm}$ (clip_d: range, -12 to $11 \mathrm{~mm}$ ) in AP, and $0.55 \pm 3.20 \mathrm{~mm}$ (clip_p: range, -10 to $9 \mathrm{~mm}$ ) and $1.35 \pm 3.63$ $\mathrm{mm}$ (clip_d: range, -17 to $10 \mathrm{~mm}$ ) in RL directions. As shown in Fig. 2, the translocation of clip after bony alignment was well correlated with diaphragm movement in 90.4\% (190/210) of all images. The mean interfractional diaphragms shift distance were $-2.8 \mathrm{~mm}$ (range, -35 to $18 \mathrm{~mm}$ ) in right diaphragm and $-4.8 \mathrm{~mm}$ (range, -39 to $18 \mathrm{~mm}$ ) in left diaphragm. The clip shift for each patient in SI direction are summarized in Fig. 3.

The shift to SI direction was most predominant in the absolute translocation of clip_p and clip_d (Fig. 2). The maximum shift was $61 \mathrm{~mm}$ in the inferior direction. The clip shift greater than $5 \mathrm{~mm}$ was observed in $26.0 \%$ in SI, 1.8\% in $A P$, and $5.4 \%$ in $R L$ directions, respectively. Moreover, the clip position errors greater than $10 \mathrm{~mm}$ was observed in 1.9\% in $\mathrm{SI}, 0.2 \%$ in $\mathrm{AP}$, and $0.2 \%$ in $\mathrm{RL}$ directions, despite respiratory control. Calculated PTV margins by VHMF were $8.7 \mathrm{~mm}$ (clip_ p) and $13.5 \mathrm{~mm}$ (clip_d) in SI, $2.4 \mathrm{~mm}$ (clip_p) and $2.4 \mathrm{~mm}$ (clip_d) in AP, and $3.9 \mathrm{~mm}$ (clip_p) and $5.0 \mathrm{~mm}$ (clip_d) in RL directions.

\section{Discussion and Conclusion}

Clinical study to compare the interfractional variation in pancreatic tumor position using bony anatomy and implanted fiducial markers showed that no fiducial shift was required after alignment to bony anatomy in 28 of 140 fractions (20\%) [7]. In this study, only 55 of 210 fractions (26.2\%) did not 
require any shift after bony alignment. Therefore, we presumed that there is substantial residual uncertainty after alignment to bony anatomy when treating hepatobiliary and pancreatic cancer patients using respiratory control. Another study of

A

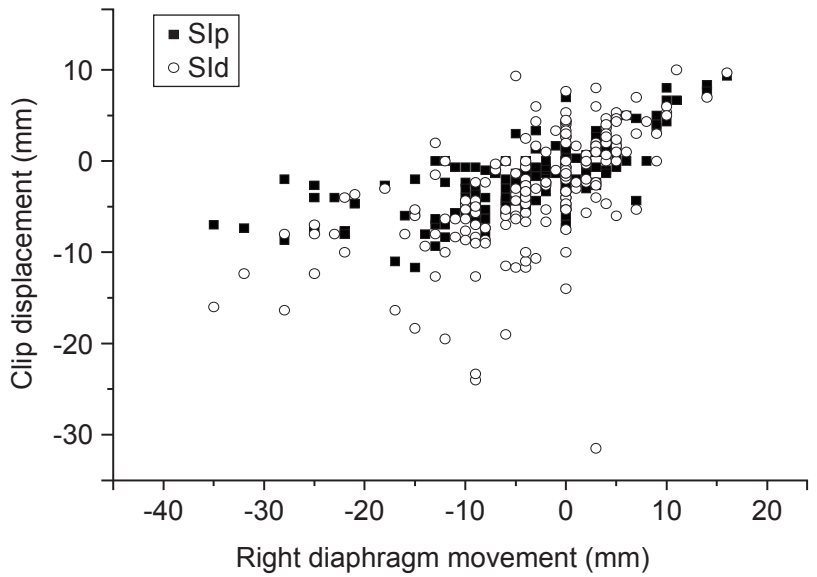

C

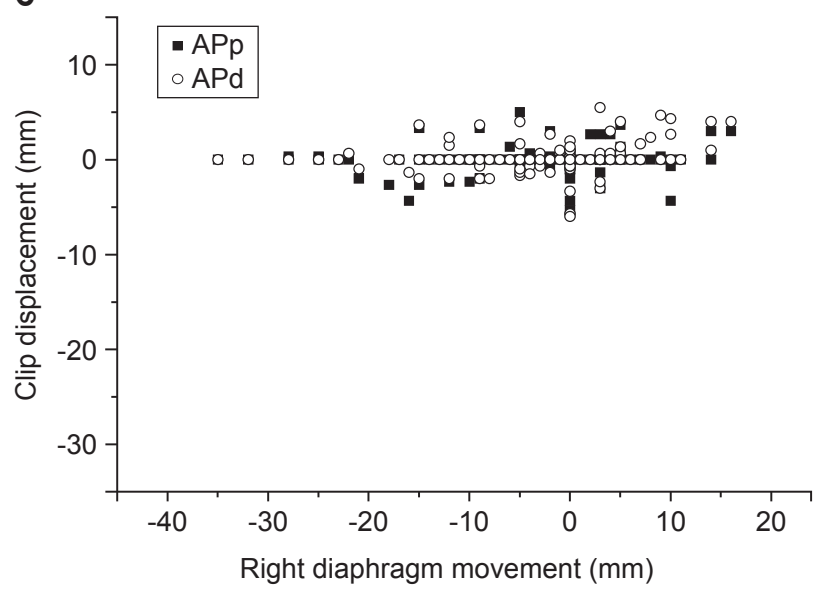

E

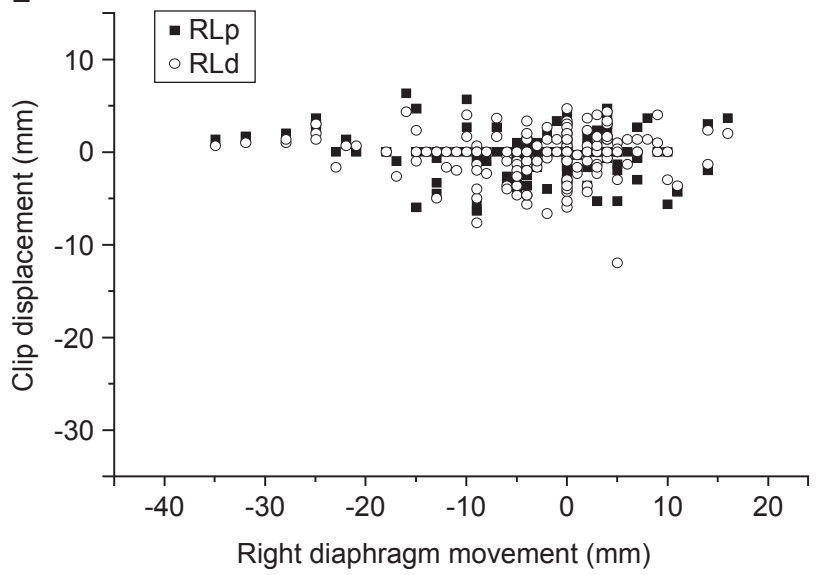

hypofractionated radiotherapy for prostate cancer patients showed that incidences of interfractional set-up error of more than $5 \mathrm{~mm}$ were $0 \%$ in the fiducial marker matching and $17.9 \%$ in the pelvic bone matching [8]. This means that the

B

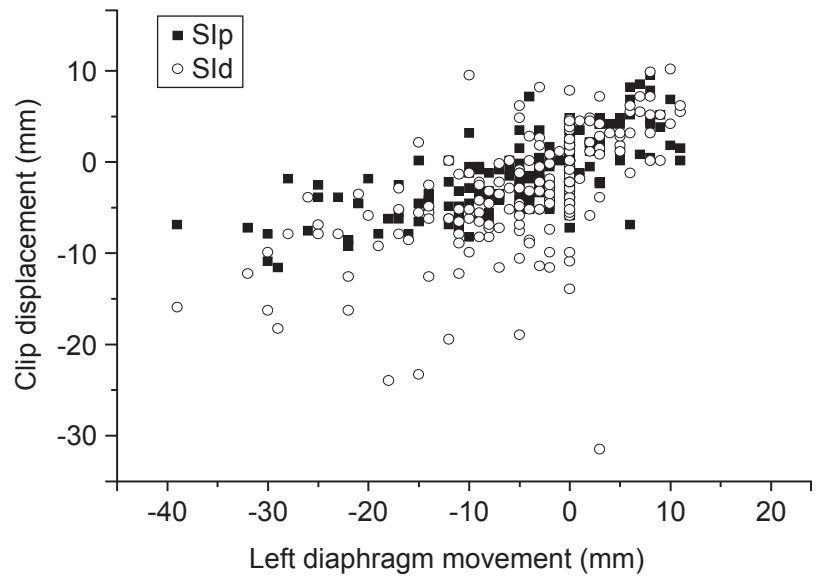

D

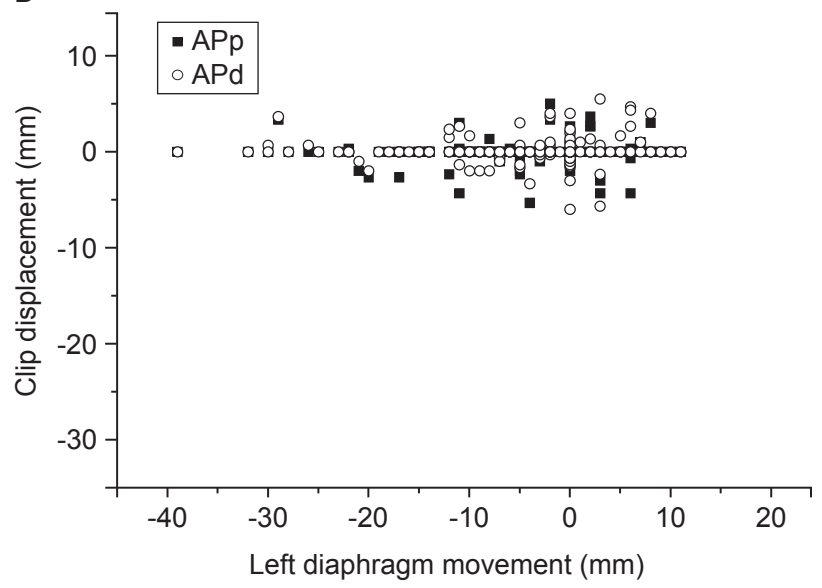

$\mathbf{F}$

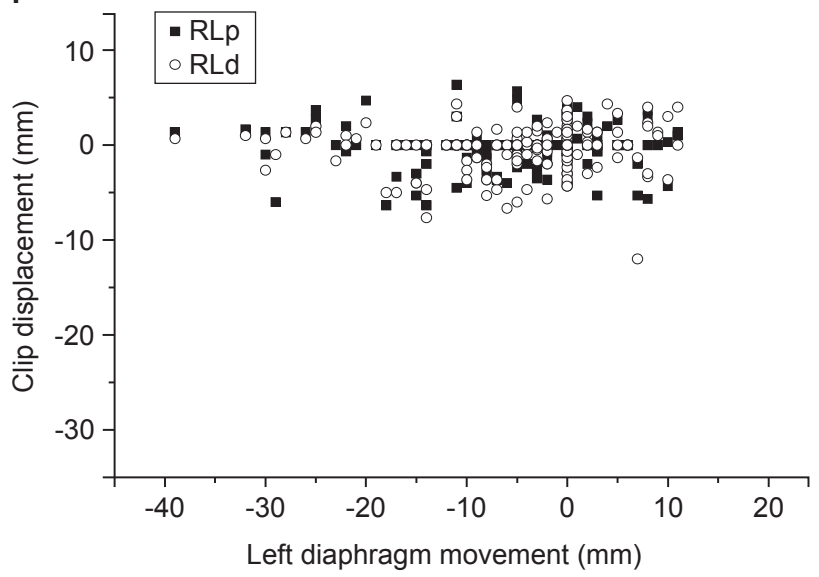

Fig. 2. Scatter plot of clip shift in $S I(A, B), A P(C, D)$, and $R L(E, F)$ directions. $S I$, superior-inferior; $A P$, anterior-posterior; $R L$, right-left; $p$, proximal clip; d, distal clip. 


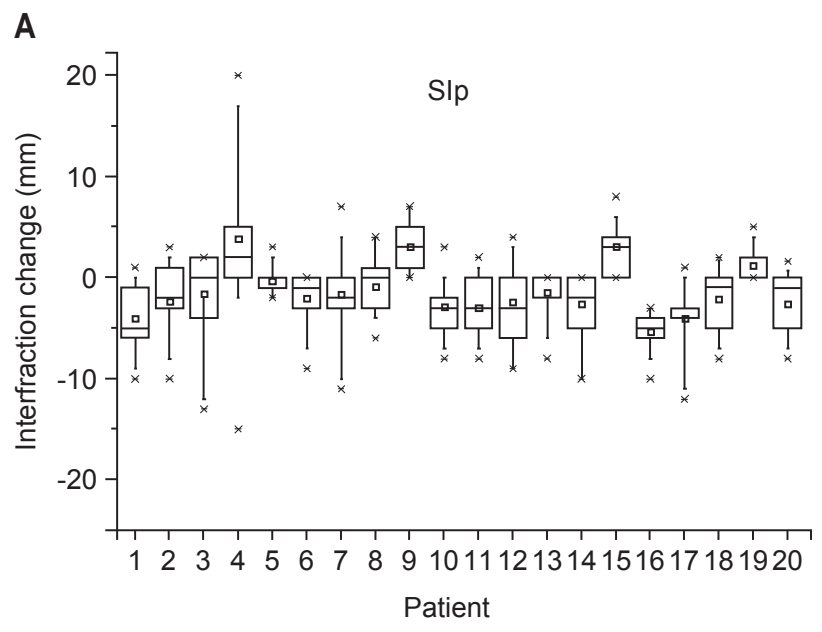

B

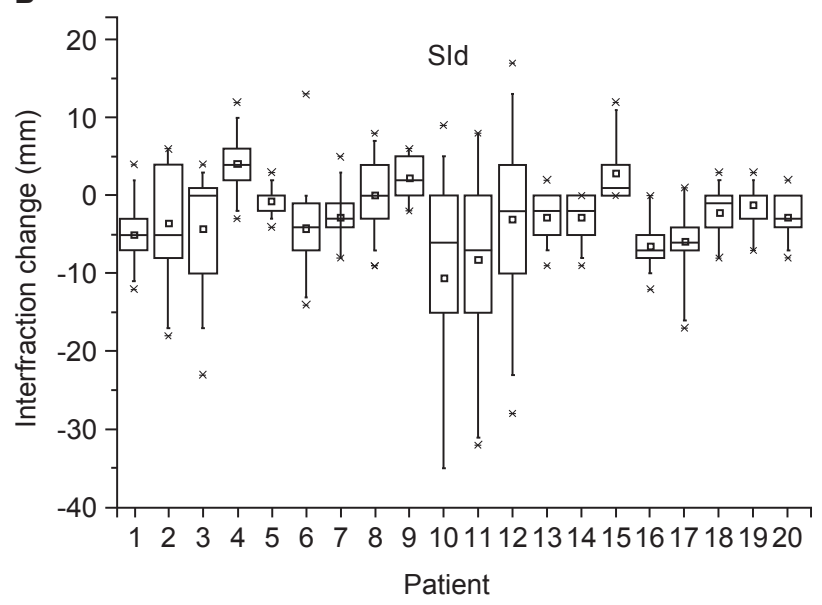

Fig. 3. (A, B) Box plot of inter-fractional clip position change in $\mathrm{SI}$ direction. SI, superior-inferior; p, proximal clip; d, distal clip.

more precise setup is achievable with the implanted fiducial marker matching compared with the pelvic bony matching.

Most patients in this study, shifting of clip in SI direction was correlated well with both diaphragm movement. The shift to SI direction was most predominant in the absolute translocation of clip_p and clip_d. These results are consistent with the study showing that the greatest pancreatic tumor motion was in the SI direction [8]. In another study, the average magnitude of the peak-to-peak motion of tumors in the abdomen was $7.4 \mathrm{~mm}$ in SI direction and $3.8 \mathrm{~mm}$ in AP direction [9]. This means that patients did not exhale enough or inhale during expiration breath-hold. More accurate information about pulmonary function, comprehension and auditory acuity of patient may elicit a result.

Delayed emptying due to postoperative change and bowel gas may induce changes in abdominal organ position. Clinical study reported that motion due to gastrointestinal distention is a significant factor for variations of the pancreas position [10]. Another study to quantify pancreas and pancreatic tumor movement due to respiratory motion showed that bowel gas also moved as a function of time, but this movement was irreproducible [11]. The clip position errors greater than 10 $\mathrm{mm}$ was observed in $1.9 \%$ in $\mathrm{SI}, 0.2 \%$ in $\mathrm{AP}$, and $0.2 \%$ in $\mathrm{RL}$ directions in present study.

One limitation of this study is the small number of patients. Due to the low incidence of target disease, our database included only 20 patients despite long periods. As a result our study have no significant clip shift according to detailed analysis such as type of operation and absolute position of clip. Another shortcoming of this work was existence of conglomerate clips. Individual clip was hard to distinguish from conglomerated clips. The nearest clip from treatment center was selected in conglomerated clips. Third, the interval between imaging and treatment have the potential of respiratory change. Respiratory change have possibility of unpredictable error. Fourth, modification was aligned to clip shifts before each treatment fraction. However, one patient's distal clips were shifted in the opposite direction. These clip displacement reflect postoperative change of intra-abdominal organ position as well as bowel gas change. Therefore, in this case, modification was aligned to proximal clip shifts. The last restrictive point, we thought, was that this study was conducted for only patients who were able to maintain an expiratory breath-hold. Although most patients were capable of maintaining the expiratory breath-hold, additional respiratory motion management such as abdominal compression, active breathing coordination, 4-dimensional $\mathrm{CT}_{\text {, }}$ and respiratory gating could decrease respiratory errors.

One hepatobiliary surgeon conducted all surgical procedures, and one radiation oncologist contoured clips and target volume in our study. Therefore, it could be considered that surgical clipping and contouring process had relative consistency. Moreover, primary tumor site, fasting status, body weight change, relieved tension according to repeated treatment and immobilization device may affect clip position change. Furthermore, interfractional organ motion, caused by hollow organ filling and weight loss or gain is difficult to estimate $[12,13]$. Patients in this study were treated with identical immobilization device fasting for 8 hours or more and daily measured body weight and abdominal circumference changed less than 5\% compare to initial state.

Matching DRR and $\mathrm{kV}$ images would take less than 5 minutes before treatment because of easily detected surgical metallic clips on $\mathrm{kV}$ verification images. Orthogonal $\mathrm{kV}$ images 
provide relatively low doses of radiation exposure and rapid treatment time compare to cone beam CT. Therefore, detecting the position of surgical clips during treatment increase safety for radiation exposure and convenience of treatment for patients and doctors.

Clinical study to quantify breathing organ motion in different breathing states showed that the median displacement in the craniocaudal direction was not exceeded $10 \mathrm{~mm}$ [14]. Therefore, clip displacement exceeding $10 \mathrm{~mm}$ is possible to consider other factors such as bowel gas, delayed gastric emptying, postoperative complication or change in the position of surrounding normal organs, despite respiratory control. Checking the position of postoperative clips before treatment may contribute to detect these errors easily. Thereafter, it requires further evaluation.

The results of our study indicate that potential exists for interfractional variations in the position of the target when treating upper abdominal tumors even after accounting for respiratory motion and daily setup error using bony anatomy. Quantitative analysis of surgical clips displacement reflect respiratory motion, setup errors and postoperative change of intra-abdominal organ position. Using of surgical metallic clips as an indicator is easily distinguished and convenient manner without invasive procedure, excessive radiation exposure and complex process. The identification of the surgical clip position may lead to a significant improvement in the accuracy of upper abdominal radiation therapy.

\section{Conflict of Interest}

No potential conflict of interest relevant to this article was reported.

\section{Acknowledgments}

This work was supported by a 2-Year Research Grant of Pusan National University.

\section{References}

1. Langen $K M$, Jones DT. Organ motion and its management. Int J Radiat Oncol Biol Phys 2001;50:265-78.

2. Perkins CL, Fox T, Elder E, Kooby DA, Staley CA 3rd, Landry J. Image-guided radiation therapy (IGRT) in gastrointestinal tumors. JOP 2006;7:372-81.

3. Bussels $B$, Goethals $L$, Feron $M$, et al. Respiration-induced movement of the upper abdominal organs: a pitfall for the three-dimensional conformal radiation treatment of pancreatic cancer. Radiother Oncol 2003;68:69-74.

4. Kim DH, Kim WT, Lee MR, et al. Influence of delayed gastric emptying in radiotherapy after a subtotal gastrectomy. J Korean Soc Ther Radiol Oncol 2009;27:194-200.

5. Oldrini G, Taste-George H, Renard-Oldrini S, et al. Implantation of fiducial markers in the liver for stereotactic body radiation therapy: feasibility and results. Diagn Interv Imaging 2015;96:589-92.

6. van Herk M, Remeijer P, Rasch C, Lebesque JV. The probability of correct target dosage: dose-population histograms for deriving treatment margins in radiotherapy. Int J Radiat Oncol Biol Phys 2000;47:1121-35.

7. Jayachandran $P$, Minn AY, Van Dam J, Norton JA, Koong AC, Chang DT. Interfractional uncertainty in the treatment of pancreatic cancer with radiation. Int J Radiat Oncol Biol Phys 2010;76:603-7.

8. Choi Y, Ahn SH, Lee HS, et al. Clinical usefulness of implanted fiducial markers for hypofractionated radiotherapy of prostate cancer. J Korean Soc Ther Radiol Oncol 2011;29:91-8.

9. Minn AY, Schellenberg $D$, Maxim $P$, et al. Pancreatic tumor motion on a single planning 4D-CT does not correlate with intrafraction tumor motion during treatment. Am J Clin Oncol 2009;32:364-8.

10. Gierga DP, Chen GT, Kung JH, Betke M, Lombardi J, Willett CG. Quantification of respiration-induced abdominal tumor motion and its impact on IMRT dose distributions. Int J Radiat Oncol Biol Phys 2004;58:1584-95.

11. Horst E, Micke O, Moustakis C, Schuck A, Schafer U, Willich NA. Conformal therapy for pancreatic cancer: variation of organ position due to gastrointestinal distention: implications for treatment planning. Radiology 2002;222:681-6.

12. Mori S, Hara R, Yanagi T, et al. Four-dimensional measurement of intrafractional respiratory motion of pancreatic tumors using a 256 multi-slice CT scanner. Radiother Oncol 2009;92:231-7.

13. Rietzel E, Rosenthal SJ, Gierga DP, Willet CG, Chen GT. Moving targets: detection and tracking of internal organ motion for treatment planning and patient set-up. Radiother Oncol 2004;73 Suppl 2:S68-72.

14. Wysocka B, Kassam Z, Lockwood G, et al. Interfraction and respiratory organ motion during conformal radiotherapy in gastric cancer. Int J Radiat Oncol Biol Phys 2010;77:53-9. 